\title{
Mechanical Systems on an almost Kähler model of a Finsler Manifold
}

\author{
Mehmet Tekkoyun * \\ Department of Econometrics, Faculty of Economics and Administration Sciences at Biga, \\ Çanakkale Onsekiz Mart University, 17200 Biga/Çanakkale, Turkey \\ Oguzhan Celik ${ }^{\dagger}$ \\ Department of Mathematics, Faculty of Science and Art, Pamukkale University, \\ Denizli, Turkey
}

November 1, 2018

\begin{abstract}
In this study, we present a new analogue of Euler-Lagrange and Hamilton equations on an almost Kähler model of a Finsler manifold. Also, we give some corollories about the related mechanical systems and equations.

Keywords: Finsler Manifold, Kähler Manifold, Kähler Einstein Manifold, Lagrangians, Hamiltonians.

MSC: 53C15, 70H03, 70H05.
\end{abstract}

\footnotetext{
*Corresponding Author. tekkoyun@comu.edu.tr

†oguzhanefe07@hotmail.com
} 


\section{Introduction}

In modern differential geometry, a suitable vector field defined on the tangent and cotangent spaces (or manifolds) which are phase-spaces of velocities and momentum of a given configuration space clarifies the dynamics of Lagrangian and Hamiltonian. If $M$ is an $n$-dimensional configuration manifold and $L(H)$ : $T M\left(T^{*} M\right) \rightarrow \mathbf{R}$ is a regular Lagrangian (Hamiltonian) function, then there is a unique vector field $\xi(X)$ on tangent (cotangent) bundle $T M\left(T^{*} M\right)$ such that dynamical formalisms and equations are given by

\begin{tabular}{cc}
\hline Dynamical Formalisms & Produced Dynamical Equations \\
\hline$i_{\xi} \Phi_{L}=d E_{L}$ & $\frac{\partial}{\partial t}\left(\frac{\partial L}{\partial \dot{q}^{i}}\right)-\frac{\partial L}{\partial q^{i}}=0$ \\
\hline$i_{X} \Phi_{H}=d H$ & $\frac{d p^{2}}{d t}=-\frac{\partial H}{\partial q^{2}}, \frac{d q^{i}}{d t}=\frac{\partial H}{\partial p^{i}}$ \\
\hline
\end{tabular}

where $\Phi_{L}\left(\Phi_{H}\right)$ indicates the (canonical) symplectic form. The triples $\left(T M, \Phi_{L}, \xi\right)$ and $\left(T^{*} M, \Phi_{H}, Z_{H}\right)$ are called Lagrangian system and Hamiltonian system on the tangent bundle $T M$ and on the cotangent bundle $T^{*} M$, respectively [1].

Anyone notes that almost Kähler models related to Finsler geometry and generalization were originally proposed by Matsumoto [2], then the Finsler idea was generalized to Lagrange geometries by Kern [3], and extended in Oproiu's works [4, 5. But, in general, almost Kähler - Finsler constructions and generalizations, with applications to modern classical and quantum physics and mechanics where performed in tenths of Vacaru's papers so that some of them are [6, 17, 8].

Really, in 9, 10, 11 being the books of R. Miron, M. Anastasiei and their other colleagues there are summarized a number of results on almost Kähler - Lagrange - Finsler/ Hamilton - Cartan geometries and generalizations with applications in mechanics.

Nevertheless, the key ideas were in Matsumoto - Kern papers and various applications related to classical and quantum gravity were considered in Vacaru's papers. There were also elaborated various Ricci flow, (non) commutative and algebroid constructions related to Finsler models and generalizations.

In [12, extrema of p-energy on constant curvature Finsler spaces was investigated. In the studies [13, 14, mechanical systems of quaternionic Kähler and paracomplex geometry were analyzed successfully. In this paper, the equations related to Finsler mechanical systems have been presented and given some corollaries.

$\mathcal{F}(M), \chi(M)$ and $\Lambda^{1}(M)$ denote the set of functions on manifold $M$, the set of vector fields on $M$ and the set of 1-forms on $M$, respectively.

\section{Finsler metric and Finsler Manifold}

In this section we recall some structures by given $[9,15$. A Finsler manifold (or Finsler space) is a pair $F^{n}=(M, F(x, y))$ where $M$ is a differential manifold of real dimension $n$ and $F: T M \rightarrow \mathbb{R}$ a scalar function which supply: 
i) $F$ is a differentiable function on the manifold $\widetilde{T M}=T M \backslash\{0\}$ and $F$ is continuous on the null section of the projection $\pi: T M \rightarrow M$.

ii) $F$ is positive function.

iii) $F$ is positively 1-homogeneous on the fibres of tangent bundle $T M$.

$i v$ ) The Hessian of $F^{2}$ with elements

$$
g_{i j}(x, y)=\frac{1}{2} \frac{\partial^{2} F^{2}}{\partial y^{i} \partial y^{j}}
$$

is positively defined on $\widetilde{T M}$. Where $g_{i j}$ is a covariant symmetric of 2 order distinguished tensor field (d-tensor field) defined on the manifold $\widetilde{T M}$.

The function $F(x, y)$ is called fundamental function and the d-tensor field $g_{i j}$ is called fundamental (or metric) tensor of the Finsler space $F^{n}=(M, F(x, y))$.

A Finsler space $F^{n}=(M, F)$ can be thought as an almost Kähler space on the manifold $\widetilde{T M}=T M \backslash\{0\}$, called the geometrical model of the Finsler space $F^{n}$. If we consider the Cartan nonlinear connection $N_{j}^{i}$ of the Finsler space $F^{n}=(M, F)$, then we can respectively define almost complex structures $\mathbf{F}$ and $\mathbf{F}^{*}$ on $T M$ and $T^{*} M$ by:

$$
\begin{gathered}
\mathbf{F}\left(\frac{\delta}{\delta x^{i}}\right)=-\frac{\partial}{\partial y^{i}}, \quad \mathbf{F}\left(\frac{\partial}{\partial y^{i}}\right)=\frac{\delta}{\delta x^{i}} \\
\mathbf{F}^{*}\left(d x^{i}\right)=-\delta y^{i}, \quad \mathbf{F}^{*}\left(\delta y^{i}\right)=d x^{i}
\end{gathered}
$$

Where the basis and dual basis are given as follows:

\begin{tabular}{ccc}
\hline$T M$ & $H T M$ & $V T M$ \\
\hline$\left(\frac{\partial}{\partial x^{2}}, \frac{\partial}{\partial y^{i}}\right)$ & $\frac{\partial}{\delta x^{i}}=\frac{\partial}{\partial x^{i}}-N_{j}^{i}(x, y) \frac{\partial}{\partial y^{i}}$ & $\frac{\partial}{\partial y^{i}}$ \\
\hline$T^{*} M$ & $H T M$ & $V T M$ \\
\hline$\left(d x^{i}, d y^{i}\right)$ & $\delta y^{i}=d x^{i}+N_{j}^{i}(x, y) d y^{i}$ & $d x^{i}$
\end{tabular}

also $\left(\frac{\partial}{\partial x^{i}}\right)^{H}=\frac{\delta}{\delta x^{i}} ;\left(d y^{i}\right)^{H}=\delta y^{i}$. It is easy to see that $\mathbf{F}$ is well defined on $\widetilde{T M}, \mathbf{F}^{2}=-I$ and it is determined only by the fundamental function $F$ of the Finsler space $F^{n}$.

Let $\left(d x^{i}, \delta y^{i}\right)$ be the dual basis of the adapted basis $\left(\frac{\delta}{\delta x^{i}}, \frac{\partial}{\partial y^{j}}\right)$. Then, the Sasaki-Matsumoto lift of the fundamental tensor $g_{i j}$ can be introduced as follows:

$$
G=g_{i j} d x^{i} \otimes d x^{j}+g_{i j} \delta y^{i} \otimes \delta y^{j} .
$$

Consequently, $G$ is a Riemann metric on $\widetilde{T M}$ determined only by the fundamental function $F$ of the Finsler space $F^{n}$ and the horizontal and vertical distributions are orthogonal with respect to it.

Theorem:

i) The pair $(G, \mathbf{F})$ is an almost Hermitian structure on $\widetilde{T M}$. 
ii) The almost symplectic 2 -form associated to the almost Hermitian structure $(G, \mathbf{F})$ is

$$
\theta=g_{i j}(x, y) \delta y^{i} \otimes d x^{j} .
$$

iii) The space $H^{2 n}=(\widetilde{T M} ; G, \mathbf{F})$ is an almost Kähler space, constructed only by means of the fundamental function $F$ of the Finsler space $F^{n}$.

Definition: The space $H^{2 n}=(\widetilde{T M} ; G, \mathbf{F})$ is called the almost Kähler model of the Finsler space $F^{n}$.

Remarking that the tensor field $\stackrel{\circ}{G}$ on $\widetilde{T M}$ given by

$$
\stackrel{\circ}{G}=g_{i j}(x, y) d x^{i} \otimes d x^{j}+\frac{a^{2}}{\|y\|^{2}} g_{i j}(x, y) \delta y^{i} \otimes \delta y^{j}, \forall(x, y) \in \widetilde{T M} .
$$

is the homogeneous lift to $\widetilde{T M}$ of the fundamental tensor field $g_{i j}$ of a Finsler space $F^{n}$,

where $a>0$ is a constant, imposed by applications (in order to preserve the physical dimensions of the components of $\stackrel{G}{G})$ and where $\|y\|^{2}=g_{i j}(x, y) y^{i} y^{j}=$ $y_{i} y^{i}=F^{2}(x, y)=2 t$ with $y_{i}=g_{i j}(x, y) y^{j}=\frac{1}{2} \frac{\partial F^{2}}{\partial y^{i}}$ such that $t$ the value of energy density in $y \in T M$.

Let us prove that the almost complex structure $\mathbf{F}$, defined by (2) does not preserve the property of homogeneity of the vector fields. Indeed, it applies the 1-homogeneous vector fields $\frac{\delta}{\delta x^{2}},(i=\overline{1, n})$ onto the 0 -homogenous vector fields $\frac{\partial}{\partial y^{i}},(i=\overline{1, n})$.

We can eliminate this $\mathbf{F}$ by defining a new kind of almost complex structure $\stackrel{\circ}{\mathbf{F}}: \chi(\widetilde{T M}) \rightarrow \chi(\widetilde{T M})$, setting :

$$
\begin{gathered}
\stackrel{\circ}{\mathbf{F}}\left(\frac{\delta}{\delta x^{i}}\right)=-\frac{\|y\|}{a} \frac{\partial}{\partial y^{i}}, \quad \stackrel{\circ}{\mathbf{F}}\left(\frac{\partial}{\partial y^{i}}\right)=\frac{a}{\|y\|} \frac{\delta}{\delta x^{i}},(i=\overline{1, n}) \\
\stackrel{\circ}{\mathbf{F}}^{*}\left(d x^{i}\right)=-\frac{\|y\|}{a} \delta y^{i}, \quad \stackrel{\circ}{\mathbf{F}}^{*}\left(\delta y^{i}\right)=\frac{a}{\|y\|} d x^{i} .
\end{gathered}
$$

\section{Euler-Lagrange Equations}

Here, we obtain Euler-Lagrange equations for quantum and classical mechanics on the almost Kähler model $\stackrel{\circ}{H}^{2 n}=(\widetilde{T M} ; \stackrel{\circ}{G}, \stackrel{\circ}{\mathbf{F}})$ of the Finsler space $F^{n}$.

Firstly, let $\stackrel{\circ}{\mathbf{F}}$ take a complex structure on the almost Kähler model $\stackrel{\circ}{H}^{2 n}$ of the Finsler space $F^{n}$, and $\left\{x^{i}, y^{i}\right\}$ be its coordinate functions.

Let semispray be the vector field $\xi$ determined by

$$
\xi=X^{i} \frac{\delta}{\delta x^{i}}+Y^{i} \frac{\partial}{\partial y^{i}}
$$


where $X^{i}=y^{i}=\dot{x}^{i}, Y^{i}=\dot{y}^{i}=\ddot{x}^{i}$ and the dot indicates the derivative with respect to time $t$.

The vector field defined by

$$
V=\stackrel{\circ}{\mathbf{F}}(\xi)=-\frac{\|y\|}{a} X^{i} \frac{\partial}{\partial y^{i}}+\frac{a}{\|y\|} Y^{i} \frac{\delta}{\delta x^{i}}
$$

is named Liouville vector field on the almost Kähler model $\stackrel{\circ}{H}^{2 n}$ of the Finsler space $F^{n}$.

The maps given by $T, P: M \rightarrow R$ such that $T=\frac{1}{2} m_{i}\left(\left(\dot{x}^{i}\right)^{2}+\left(\dot{y}^{i}\right)^{2}\right), P=$ $m_{i} g h$ are said to be the kinetic energy and the potential energy of the system, respectively. Here $m_{i}, g$ and $h$ stand for mass of a mechanical system having $m$ particles, the gravity acceleration and distance to the origin of a mechanical system on the almost Kähler model $\stackrel{\circ}{H}^{2 n}$ of the Finsler space $F^{n}$, respectively.

Then $L: M \rightarrow R$ is a map that satisfies the conditions; i) $L=T-P$ is a Lagrangian function, ii) the function determined by $E_{L}=V(L)-L$, is energy function.

The function $i_{\mathbf{F}}$ induced by $\stackrel{\circ}{\mathbf{F}}$ and denoted by

$$
i_{\mathbf{F}} \omega\left(X_{1}, X_{2}, \ldots, X_{r}\right)=\sum_{i=1}^{r} \omega\left(X_{1}, \ldots, \stackrel{\circ}{\mathbf{F}} X_{i}, \ldots, X_{r}\right),
$$

is called vertical derivation, where $\omega \in \wedge^{r} M, X_{i} \in \chi(M)$.

The vertical differentiation $d_{\mathbf{F}}$ is given by

$$
d_{\stackrel{\mathbf{F}}{\circ}}=\left[i_{\mathbf{F}}, d\right]=i_{\mathbf{F}} d-d i_{\mathbf{F}}
$$

where $d$ is the usual exterior derivation.

For $\stackrel{\circ}{\mathbf{F}}$, the closed Kähler form is the closed 2-form given by $\Phi_{L}=-d d_{\mathbf{F}} L$ such that

$$
d_{\mathbf{F}}=-\frac{\|y\|}{a} \frac{\partial}{\partial y^{i}} d x^{i}+\frac{a}{\|y\|} \frac{\delta}{\delta x^{i}} \delta y^{i}: \mathcal{F}(\widetilde{T M}) \rightarrow \wedge \widetilde{T M}
$$

Then we have

$$
\begin{aligned}
\Phi_{L}= & -d d_{\mathbf{F}} L=\frac{\|y\|}{a} \frac{\delta}{\delta x^{j}}\left(\frac{\partial L}{\partial y^{i}}\right) d x^{j} \wedge d x^{i}-\frac{a}{\|y\|} \frac{\delta^{2} L}{\delta x^{j} \delta x^{i}} d x^{j} \wedge \delta y^{i} \\
& +\frac{\|y\|}{a} \frac{\partial^{2} L}{\partial y^{j} \partial y^{i}} \delta y^{j} \wedge d x^{i}-\frac{a}{\|y\|} \frac{\partial}{\partial y^{j}}\left(\frac{\delta L}{\delta x^{i}}\right) \delta y^{j} \wedge \delta y^{i}
\end{aligned}
$$

Let $\xi$ be the second order differential equation (semispray) given by (9). Then we calculate

$$
\begin{aligned}
& i_{\xi} \Phi_{L}=\Phi_{L}(\xi)=\frac{\|y\|}{a} \frac{\delta}{\delta x^{j}}\left(\frac{\partial L}{\partial y^{i}}\right) X^{i} \delta_{i}^{j} d x^{i}-\frac{\|y\|}{a} \frac{\delta}{\delta x^{j}}\left(\frac{\partial L}{\partial y^{i}}\right) X^{i} d x^{j} \\
& -\frac{\|y\|}{a} \frac{\partial^{2} L}{\partial y^{j} L y^{i}} X^{i} \delta y^{j}-\frac{a}{\|y\|} \frac{\delta^{2} L}{\delta x^{j} \delta x^{i}} X^{i} \delta_{i}^{j} \delta y^{i}+\frac{\|y\|}{a} \frac{\partial^{2} L}{\partial y^{j} \partial y^{i}} Y^{i} \delta_{i}^{j} d x^{i} \\
& +\frac{a}{\|y\|} \frac{\delta^{2} L}{\delta x^{j} \delta x^{i}} Y^{i} \delta y^{j}-\frac{a}{\|y\|} \frac{\partial}{\partial y^{j}}\left(\frac{\delta L}{\delta x^{i}}\right) Y^{i} \delta_{i}^{j} \delta y^{i}+\frac{a}{\|y\|} \frac{\partial}{\partial y^{j}}\left(\frac{\delta L}{\delta x^{i}}\right) Y^{i} \delta y^{j}
\end{aligned}
$$


Energy function and its differential are

$$
E_{L}=V(L)-L=-\frac{\|y\|}{a} X^{i} \frac{\partial L}{\partial y^{i}}+\frac{a}{\|y\|} Y^{i} \frac{\delta L}{\delta x^{i}}-L
$$

and

$$
\begin{gathered}
d E_{L}=-\frac{\|y\|}{a} X^{i} \frac{\delta}{\delta x^{j}}\left(\frac{\partial L}{\partial y^{i}}\right) d x^{j}-\frac{\|y\|}{a} X^{i} \frac{\partial^{2} L}{\partial y^{j} \partial y^{i}} \delta y^{j} \\
+\frac{a}{\|y\|} Y^{i} \frac{\delta^{2} L}{\delta x^{j} \delta x^{i}} d x^{j}+\frac{a}{\|y\|} Y^{i} \frac{\partial}{\partial y^{j}}\left(\frac{\delta L}{\delta x^{i}}\right) \delta y^{j}-\frac{\delta L}{\delta x^{j}} d x^{j}-\frac{\partial L}{\partial y^{j}} \delta y^{j}
\end{gathered}
$$

Using $i_{\xi} \Phi_{L}=d E_{L}$, we find the expressions as follows:

$$
\begin{gathered}
\frac{\|y\|}{a}\left[X^{i} \frac{\delta}{\delta x^{i}}+Y^{i} \frac{\partial}{\partial y^{i}}\right]\left(\frac{\partial L}{\partial y^{i}}\right) d x^{i}+\frac{\delta L}{\delta x^{i}} d x^{i} \\
-\frac{a}{\|y\|}\left[X^{i} \frac{\delta}{\delta x^{i}}+Y^{i} \frac{\partial}{\partial y^{i}}\right]\left(\frac{\delta L}{\delta x^{i}}\right) \delta y^{i}+\frac{\partial L}{\partial y^{i}} \delta y^{i}=0
\end{gathered}
$$

and

$$
\frac{\|y\|}{a} \xi\left(\frac{\partial L}{\partial y^{i}}\right) d x^{i}+\frac{\delta L}{\delta x^{i}} d x^{i}-\frac{a}{\|y\|} \xi\left(\frac{\delta L}{\delta x^{i}}\right) \delta y^{i}+\frac{\partial L}{\partial y^{i}} \delta y^{i}=0
$$

By means of integral curve $\xi(t)=\dot{\alpha}(t)$, we have the equations

$$
\frac{\|y\|}{a} \frac{d}{d t}\left(\frac{\partial L}{\partial y^{i}}\right)+\frac{\delta L}{\delta x^{i}}=0, \frac{a}{\|y\|} \frac{d}{d t}\left(\frac{\delta L}{\delta x^{i}}\right)-\frac{\partial L}{\partial y^{i}}=0
$$

such that the equations calculated in (10) are named Euler-Lagrange equations constructed on the almost Kähler model $\stackrel{\circ}{H}^{2 n}$ of the Finsler space $F^{n}$ and thus the triple $\left(\stackrel{\circ}{H}^{2 n}, \Phi_{L}, \xi\right)$ is called a mechanical system on the almost Kähler model $\stackrel{\circ}{H}^{2 n}$ of the Finsler space $F^{n}$.

\section{Hamilton Equations}

Here, we present Hamilton equations and Hamiltonian mechanical systems for quantum and classical mechanics constructed on the almost Kähler model $\stackrel{\circ}{H}^{* 2 n}=$ $\left(\widetilde{T M} ; \stackrel{\circ}{G}, \stackrel{\circ}{*}^{*}\right)$ of the Finsler space $F^{n}$.

Firstly let set a 1-form

$$
\omega=\frac{a^{2}}{\|y\|^{2}} x^{i} d x^{i}+y^{i} \delta y^{i} .
$$

Then we have the Liouville form

$$
\lambda=\stackrel{\circ}{\mathbf{F}}^{*}(\omega)=-\frac{a}{\|y\|} x^{i} \delta y^{i}+\frac{a}{\|y\|} y^{i} d x^{i} .
$$

and the closed form

$$
\phi=-d(\lambda)=\frac{a}{\|y\|} d x^{i} \wedge \delta y^{i}
$$


Take Hamiltonian vector field as follows:

$$
X=X^{i} \frac{\partial}{\partial x^{i}}+Y^{i} \frac{\delta}{\delta y^{i}},
$$

Then we find

$$
i_{X} \Phi_{H}=\Phi_{H}(X)=\frac{a}{\|y\|} X^{i} \delta y^{i}-\frac{a}{\|y\|} Y^{i} d x^{i}
$$

and

$$
d H=\frac{\partial H}{\partial x^{i}} d x^{i}+\frac{\delta H}{\delta y^{i}} \delta y^{i} .
$$

By means of $i_{X} \Phi_{H}=d H$, the Hamiltonian vector field is found as follows:

$$
X=\frac{\|y\|}{a} \frac{\delta H}{\delta y^{i}} \frac{\partial}{\partial x^{i}}-\frac{\|y\|}{a} \frac{\partial H}{\partial x^{i}} \frac{\delta}{\delta y^{i}} .
$$

Assume that a curve

$$
\alpha: I \subset \mathbf{R} \rightarrow \widetilde{T^{*} M}
$$

be an integral curve of the Hamiltonian vector field $X$, i.e.,

$$
X(\alpha(t))=\dot{\alpha}(t), t \in I .
$$

In the local coordinates, it is obtained that

$$
\alpha(t)=\left(x^{i}, y^{i}\right)
$$

and

$$
\dot{\alpha}(t)=\frac{d x^{i}}{d t} \frac{\partial}{\partial x^{i}}+\frac{d y^{i}}{d t} \frac{\delta}{\delta y^{i}} .
$$

Taking (12), if we equal (11) and (13), it holds

$$
\frac{d x^{i}}{d t}=\frac{\|y\|}{a} \frac{\delta H}{\delta y^{i}}, \quad \frac{d y^{i}}{d t}=-\frac{\|y\|}{a} \frac{\partial H}{\partial x^{i}}
$$

Hence, the equations introduced in (14) are named Hamilton equations on the almost Kähler model $\stackrel{\circ}{H}^{* 2 n}$ of Finsler manifold $F^{n}$ and then the triple $\left(\stackrel{\circ}{H}^{* 2 n}, \Phi_{H}, X\right)$ is said to be a Hamiltonian mechanical system on the almost Kähler model $\stackrel{\leftrightarrow}{H}^{* 2 n}$ of Finsler manifold $F^{n}$.

\section{Conclusion}

Here, we may give the following corollaries considering the above structures and found equations by means of 4 .

Corollary 1: The almost complex structure $\mathbf{F}\left(\mathbf{F}^{*}\right)$ on $T M$ is integrable if $(M, G)$ has constant sectional curvature $c$ and the function $v$ is given by

$v=\frac{c-u u^{\prime}}{2 t u^{\prime}-u}$, where $u>0, u+2 t v>0$ for all $t \geq 0$. 
Corollary 2: Assume that $(M, G)$ has constant negative curvature $c$. Then $\stackrel{\circ}{H}^{2 n}\left(\stackrel{\circ}{H}^{* 2 n}\right)$ with the following $u$ and $v$ is a Kähler Einstein manifold. Where $A>0, t \geq 0, u=A+\sqrt{A^{2}-2 c t}>0, v=\frac{1}{2 t}\left(A-\frac{4 c t}{A}-\sqrt{A^{2}-2 c t}\right)>0$. So, the equations found in (10) (14)) are named Euler-Lagrange (Hamilton) equations constructed on the almost Kähler Einstein model $\stackrel{\circ}{H}^{2 n}\left(\stackrel{H}{H}^{* 2 n}\right)$ of constant negative curvature $c$ of the Finsler space $F^{n}$

Corollary 3: Assume that $(M, G)$ has constant positive curvature $c$. Then the tube around the zero section in $T M\left(T^{*} M\right)$, defined by the condition $\|y\|^{2}=$ $g_{i k} y^{i} y^{k}=2 t<\frac{A^{2}}{c}$ has a structure of Kähler Einstein manifold, if the functions $u, v$ are given as follows:

$A>0, u=A+\sqrt{A^{2}-2 c t}, v=\frac{1}{2 t}\left(A-\frac{4 c t}{A}-\sqrt{A^{2}-2 c t}\right), 0 \leq t<\frac{A^{2}}{c}$. Hence, the equations found in (10) (14)) are named Euler-Lagrange (Hamilton) equations constructed on the almost Kähler Einstein model $\stackrel{\circ}{H}^{2 n}\left(\stackrel{(H}{H}^{* 2 n}\right)$ of constant positive curvature $c$ of the Finsler space $F^{n}$.

Acknowledgement: I would like to express my gratitude to Vacaru who gave me his valuable comments and discussions to complete of this study.

\section{References}

[1] M. De Leon, P.R. Rodrigues, Methods of Differential Geometry in Analytical Mechanics, North-Holland Mathematics Studies, Vol.152, Elsevier, Amsterdam, 1989.

[2] M. Matsumoto, Foundations of Finsler Geometry and Special Finsler Spaces (Kaisisha: Shingaken, Japan, 1986).

[3] J. Kern, Lagrange Geometry, Archiv der Mathematik (Basel) 25 (1974) 438-443.

[4] V. Oproiu, Some properties of the tangent bundle related to the Finsler geometry, Proced. Nat. Sem. Finsler Spaces, Brasov, 1980, 195-207.

[5] V. Oproiu, A Riemannian structure in Lagrange geometry, Rend. Sem. Fac. Sci. Univ. Cagliari, 55 (2),(1985), 1-20.

[6] D. Baleanu, S. I. Vacaru, Fractional Almost Kähler - Lagrange Geometry, arXiv:1006.5535v3 [math-ph].

[7] S. I. Vacaru, Lagrange-Ricci Flows and Evolution of Geometric Mechanics and Analogous Gravity on Lie Algebroids, arXiv:1108.4333v2 [math-ph].

[8] S. I. Vacaru, Finsler and Lagrange Geometries in Einstein and String Gravity, arXiv:0801.4958v1 [gr-qc].

[9] R. Miron, D. Hrimiuc, H. Shimada, S. V. Sabau, The Geometry of Hamilton and Lagrange Spaces, Hingham, MA, USA: Kluwer Academic Publishers, 2001.P.L. 
[10] P. L. Antonelli and R.Miron, Lagrange and Finsler Geometry. Applications to Physics and Biology, Kluwer Academic Publishers, FTPH 76, 1996.

[11] R.Miron and M.Anastasiei, Vector Bundles. Lagrange spaces. Applications to the Theory of Relativity, Balkan Press, Bucuresti, 1997.

[12] C. Udrişte, M. Neagu, Extrema of p-Energy on Constant Curvature Finsler Spaces, Differential Geometry-Dynamical Systems (DGDS), Vol: 1., No:1, (1999) 10-19.

[13] M. Tekkoyun, On Para-Euler Lagrange and Para- Hamiltonian Equations,Physics Letters A, Vol. 340, (2005) 7-11.

[14] M. Tekkoyun, Y. Yayli, Mechanical Systems on Generalized on Quaternionic Kähler Manifolds,, International Journal of Geometric Methods in Modern Physics (IJGMMP), Vol. 8, No. 7 (2011) 1419-1431.

[15] V. Oproiu, A Kähler Einstein Structure on the Tangent Bundle of a Space Form, IJMMS 25:3 (2001)183-195. 